\title{
Usefulness of for BI-RAD category 4 or higher lesions
}

\author{
Jun Suk Byun', Hyeon Hwa Oh', Hye Yoon Lee', Keum Won Kim², Jin Suk Kim³, Dae Sung Yoon' \\ Departments of 'Surgery, ${ }^{2}$ Radiology, and ${ }^{3}$ Nuclear Medicine, Konyang University, Konyang University Hospital, Daejeon, Korea
}

Purpose: As an alternative to core-needle biopsy in confirming the diagnosis of breast cancer, the usefulness of ${ }^{99 \mathrm{~m} T c-s e s t a m i b i}$ scintimammography (MIBI scan) has been rarely reported. Thus, we aimed to evaluate a direct comparison between general diagnostic modalities and breast MIBI scan, which may be revealed as a potential diagnostic alternative.

Methods: In a retrospective study, 301 patients with 801 lesions, who underwent breast MIBI scan, ultrasonography, and mammography simultaneously between January 2013 and February 2015, were reviewed. All data were analyzed by McNemar and Kappa test for statistical significance.

Results: Mean age was $49.2 \pm 9.37$ years old (range from 26 to 85 years old). Results of Breast MIBI scan were divided into three categories: 236 positive intensity uptakes (29.5\%), 565 negative intensity uptakes (70.5\%), and 67 suspicious abnormal intensity uptakes (8.4\%). Pathologic reports were also allocated into four subgroups: 122 invasive cancers (15.2\%), 44 non-invasive cancers (5.5\%), 194 proliferative benign lesions (24.2\%), and 441 non-proliferative benign lesions (55.1\%). The sensitivity, specificity, positive predictive value, and negative predictive value of MIBI scan were $83.5 \%, 55.6 \%, 59.1 \%$, and $95.2 \%$, respectively. Specificity of MIBI scan plus general diagnostic modalities increased dramatically, up to $85.2 \%$, compared to general modalities (6.8\%).

Conclusion: In general diagnostic modalities, such as mammography and ultrasound, BI-RAD category 4 or higher lesions were performed by needle biopsy rather than observation. The outstanding specificity and negative predictive value of MIBI scan provided confident results on non-proliferative benign lesions. MIBI scan may offer an alternative diagnostic tool for "invasive" biopsy procedures.

Keywords: Breast cancer, Scintimammography, BI-RADS category 4

\section{INTRODUCTION}

Breast cancer has been one of the most common cancers for women in the Western Hemisphere. In recent decades, Korean women have also become more susceptible to breast cancer, and its incidence has been increasing up to approximately $40 \%$ [1]. It is a very heterogeneous disease entity that is more difficult to diagnose than to treat. Therefore, early diagnosis and treatment can improve the prognosis of breast cancer. The diagnosis of patients with breast cancer has used various methods including clinical examination

Received: Nov 3, 2016 Accepted: Jun 13, 2017

Correspondence to: Dae Sung Yoon

Department of Surgery, Center of Breast and Thyroid Cancers, Konyang University Hospital, 158 Gwanjeodong-ro, Seo-gu, Daejeon 35365, Korea Tel: +82-42-600-8956, Fax: +82-42-524-8956

E-mail: dsyoonmd@kyuh.ac.kr

Copyright $($ Korean Society of Surgical Oncology

This is an Open Access article distributed under the terms of the Creative Commons Attribution Non-Commercial License (http://creativecommons.org/licenses/by-nc/4.0) which permits unrestricted non-commercial use, distribution, and reproduction in any medium, provided the original work is properly cited. and imaging modalities such as ultrasonography (US), mammography (MMG), and magnetic resonance imaging (MRI). Although all these modalities can be used to detect breast lesions, and the sensitivity has been reported $96 \%$ in other previous studies, each imaging modality does have its own downside [2]. The sensitivity of breast mammography decreases in patients with dense breasts or who received estrogen treatment. Ultrasonography has not been able to detect microcalcifications. Breast MRI might raise the risk of unnecessary biopsy or operation due to higher rates of false positivity [3-6].

In 1994, Khalkhali et al. [7] reported first that breast scintimammography using ${ }^{99 m}$ Tc-sestamibi could be used as a breast screening modality. It was a physiological approach toward the breast lesions rather than an anatomical view. The malignant cells usually had higher vascularity and mitochondrial activities, which was more prone to uptake a large quantity of injected isotopes compared to the normal cells $[8,9]$. The authors reported that the sensitivity, specificity, positive predictive value, and negative predictive value were $95.8 \%, 86.8 \%, 82.1 \%$, and $97.1 \%$, respectively [7]. Since 
then, technological advancements and progress have been made in high-resolution breast-specific gamma imaging with ${ }^{99 m} \mathrm{Tc}$-sestamibi scintimammography (MIBI scan). The sensitivity and specificity of breast-specific gamma imaging have been reported as $89 \%-96 \%, 60 \%-90 \%$, respectively [10-14].

The usefulness of MIBI scan has been continuously reported as an adjunct modality for detecting breast cancers as an alternative to core-needle biopsy in confirming an initial diagnosis [15,16]. Although most previous studies were oriented to recurrence or in conjunction with other modalities, there have only been a limited number of reports directly comparing MIBI scan with other modalities.

The diagnostic value of MIBI scan in breast cancer and its correlation with conventional diagnostic images may be necessary to recapture the current agenda of breast cancer diagnostic modalities, and this study has focused on those factors of MIBI scan and evaluated the very value thereof. In this study, patients without symptoms or signs were not included, as its diagnostic values were not for simple screening among the general populous. Thus, we aimed to evaluate the usefulness breast MIBI scan to reveal it as a potential diagnostic alternative.

\section{METHODS}

\section{Participants}

In a retrospective study, 645 patients with breast lesions, who underwent MIBI scan between January 2013 and February 2015, were enrolled. A total of 301 patients with 801 lesions, who underwent breast MIBI scan, ultrasonography, and mammography simultaneously, between January 2013 and February 2015, were reviewed. Three hundred forty-four patients were excluded due to incompletion of study modalities and loss of follow-up. We performed MIBI scans on the follows cases: (a) patients who were diagnosed with malignancy prior to surgery, (b) patients who were followed up after cancer surgery, (c) patients with lesions that could not be evaluated by breast ultrasonography or mammography, (d) patients with multiple benign lesions, and (e) patients with suspicious lesions who refused biopsy initially (and eventually agreed to the biopsy). We analyzed the MIBI scan results with those of breast ultrasonography, mammography, and pathology per lesion.

\section{Image modalities}

For MIBI scan, patients were injected with $30 \mathrm{mCi}(1110 \mathrm{MBq})$ of technetium-99m sestamibi (Dong-A Pharmaceutical, Seoul, Korea) into an arm vein contralateral to the breast of interest. A dorsalis pedis vein was used if no suitable arm vein was found. Imaging was begun immediately after injection of the isotope. Cranio- caudal and mediolateral views were performed of both breasts with approximately 10 minutes per view (total time, 40 minutes). Images were obtained with a high-resolution, small field-of-view, breast-specific gamma camera (Dilon 6800 Gamma Camera, Dilon Technologies, Newport News, VA). Mammography was performed with a Senographe DS (GE Medical Systems, Milwaukee, WI, USA), and craniocaudal and mediolateral oblique images of the breasts were obtained bilaterally. Ultrasonography was performed with an HDI 5000 scanner (Philips Medical Systems, Bothell, WA, USA) or an IU 22 scanner (Philips Medical Systems).

\section{Image evaluation}

All images were interpreted by three individual radiologists who were dedicated specifically to the breast lesions, and reported according to the Breast Imaging and Reporting Data System, BIRADS. Category 0, 4, 5, 6 were considered as positive findings. A category 0 lesion was considered a positive finding as it meant that that lesion needed further study to exclude the possibility of malignancy. Therefore, all the images were classified as either negative, no further work-up recommended; or positive, further work-up such as additional imaging or biopsy recommended.

\section{Statistical analysis}

Data have been categorized by all lesions with biopsy confirmed along with MIBI scan results. Data were collected retrospectively and stored per-lesion in a Microsoft Excel 2010 program (Microsoft Corporation, Redmond, WA, USA). Statistical analysis was performed using the SPSS statistical software package (ver. 18, SPSS Inc., Chicago, IL, USA). All data were analyzed by McNemar and Kappa test for statistical significance. P-value less than 0.05 was considered to be statistically significant.

\section{RESULTS}

The mean age was $49.2 \pm 9.37$ years old (range from 26 to 85 years old). Of the 801 breast lesions, 166 lesions (20.7\%) proved to be malignant lesions whereas 635 lesions $(79.3 \%)$ proved to be benign lesions (Table 1). The detection results of breast MIBI scan were divided into three categories: 236 positive intensity uptakes (29.5\%), 565 negative intensity uptakes (70.5\%), and 67 suspicious abnormal intensity uptakes (8.4\%). The pathologic reports were also allocated into four subgroups: 122 invasive cancers (15.2\%), 44 non-invasive cancers (5.5\%), 194 proliferative benign lesions (24.2\%), and 441 non-proliferative benign lesions (55.1\%).

The diagnostic sensitivity, specificity, positive predictive value, and negative predictive value were $83.5 \%, 84.9 \%, 59.1 \%$, and $95.2 \%$, respectively. In comparison to the general modalities, ultra- 
Table 1. Patients' demographics

\begin{tabular}{lc}
\hline Variables & Patients $(\mathrm{n}, \%)$ \\
\hline Mean age (range) & $49.25 \pm(26-85)$ \\
No. of cases (patient) & $801(301)$ \\
Benign & \\
Proliferative lesions & $194(24.21)$ \\
Non-proliferative lesions & $441(55.05)$ \\
Total & $635(79.27)$ \\
Malignancy & \\
Invasive lesions & $122(15.23)$ \\
Non-invasive lesions & $44(5.493)$ \\
Total & $166(20.72)$ \\
\hline
\end{tabular}

Table 2. Comparative results of all diagnostic modalities

\begin{tabular}{lcccc}
\hline Variables & MIBI & MMG & US & P-value \\
\hline Sensitivity & 83.7 & 92.2 & 95.2 & $<0.001$ \\
Specificity & 84.9 & 55.6 & 13.2 & $<0.001$ \\
PPV & 59.1 & 35.2 & 22.3 & $<0.001$ \\
NPV & 95.2 & 96.4 & 91.3 & $<0.001$ \\
\hline
\end{tabular}

Values are presented as percentage.

MIBI, ${ }^{99 m}$ Tc-methoxyisobutyl-isonitrile scintimammography; MMG, mammography; US, ultrasonography; PPV, positive predictive value; NPV, negative predictive value.

${ }^{\text {a) By McNemar. }}$

Table 3. Comparative results of combining MIBI scan with other general modalities

\begin{tabular}{lccc}
\hline Variables & US/MMG & US/MMG+MIBI & P-value $^{\text {a) }}$ \\
\hline Sensitivity & 99.4 & 83.1 & $<0.001$ \\
Specificity & 6.8 & 85.2 & $<0.001$ \\
PPV & 21.8 & 59.5 & $<0.001$ \\
NPV & 97.7 & 95.1 & $<0.001$ \\
\hline
\end{tabular}

Values are presented as percentage.

MIBI, ${ }^{99 m}$ Tc-methoxyisobutyl-isonitrile scintimammography; US, ultrasonography; MMG, mammography; PPV, positive predictive value; NPV, negative predictive value.

a)By McNemar.

sonography and mammography, the specificity and negative predictive values of MIBI scan displayed 'better' diagnostic power by $71 \%-21 \%$ and $4 \%$, respectively (Table 2 ). Values for all the modalities showed statistical significance so that they could be compared directly. The general screening modalities plus the MIBI scan have greatly improved specificity and positive predictive values by at least 30\% (Table 3). Especially, the specificity of MIBI scan plus general diagnostic modalities dramatically increased up to $85.2 \%$ compared to general modalities (6.8\%).

There were 92 lesions assigned to BI-RADS category 3 or less in the breast ultrasonography, but only 8 lesions (8.69\%) showed pos-
Table 4. Pathology of MIBI scans in false-positive results

\begin{tabular}{lr}
\hline Pathology & No. \\
\hline Proliferative lesions & 54 \\
Fibroadenoma & 5 \\
Adenosis & 7 \\
Intraductal papilloma & 2 \\
Atypical ductal hyperplasia & 7 \\
Phyllodes tumor & 3 \\
Non proliferative lesions & 42 \\
Fibrocystic change & 30 \\
Duct ectasia & 4 \\
Stromal fibrosis & 14 \\
Inflammation & 2 \\
Vasculitis & 4 \\
Total & 96 \\
\hline
\end{tabular}

MIBI scan, ${ }^{99 m}$ Tc-methoxyisobutyl-isonitrile scintimammography.

Table 5. Comparison of MIBI scans and biopsy results in axillary lesions

\begin{tabular}{lcc}
\hline Overall results & MIBI scan & Biopsy results (\%) \\
\hline Positive & 33 & $22(66.6)$ \\
Negative & 768 & NA \\
\hline
\end{tabular}

Most negative lymph nodes were not found through biopsy.

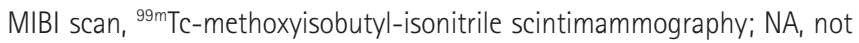
available.

itive intensity uptakes in the MIBI scan whereas 84 lesions (91.3\%) were negative intensity uptakes (Table 4). There were 435 lesions detected in the breast mammography, but only 192 lesions (44.1\%) showed positive intensity uptakes in the MIBI scan whereas 243 lesions (55.8\%) were negative intensity uptakes.

There were 33 axillary lesions positive intensity uptakes in the MIBI scan whereas 768 lesions were negative intensity uptakes. Among those 33 axillary positive lesions, 22 lesions (66.6\%) were related to the malignant lesions. Although there was a definite focal intensity uptake for the axillary area in the MIBI scan, $66.6 \%$ of the detection rate was not sufficient to assure its results (Table 5).

\section{DISCUSSION}

The purpose of this study was to compare the diagnostic results of MIBI scan, ultrasonography, and mammography in breast lesions and to analyze the final pathologic results. MIBI scan had a sensitivity and specificity of $83.7 \%$ and $84.9 \%$, respectively, and had a significantly greater specificity and negative predictive value compared with mammography and ultrasonography $(\mathrm{P}<0.001)$. It also had a greater positive predictive value compared with mammography and ultrasonography $(\mathrm{P}<0.001)$, but the positive pre- 

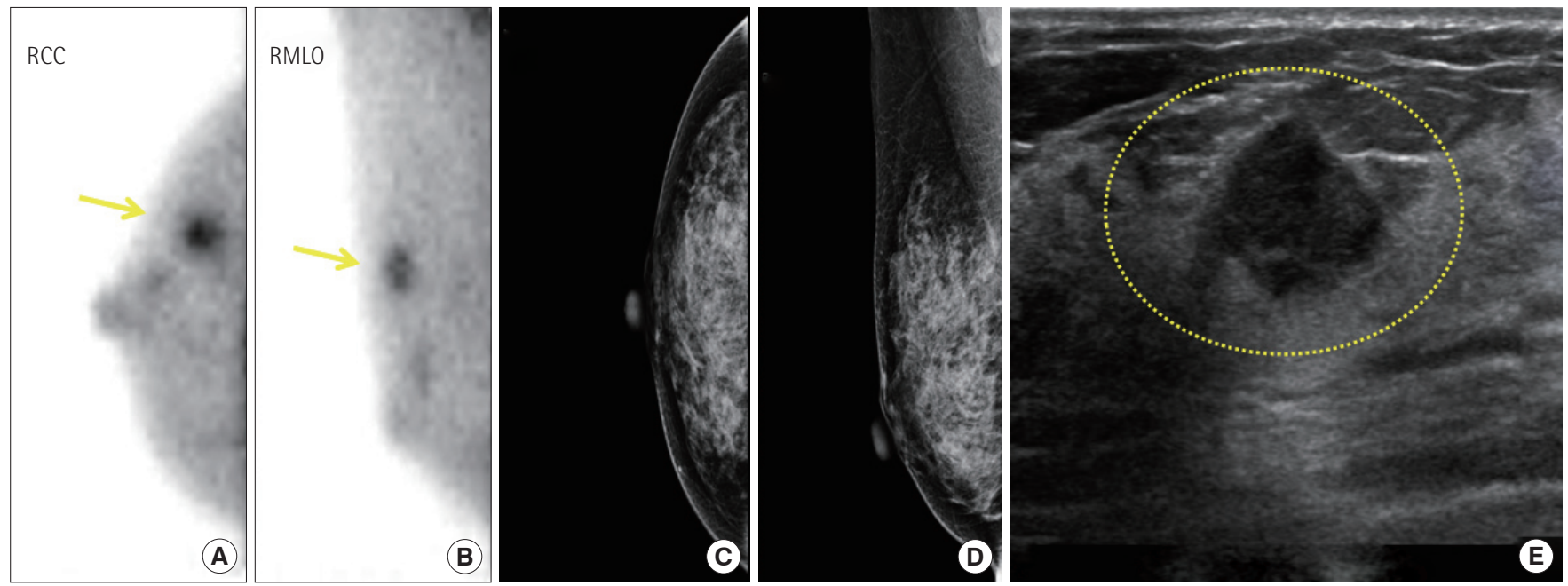

Fig. 1. MIBI scan positive result (Malignant lesion). Breast images of 42-year-old woman with a biopsy confirmed $4 \mathrm{~cm}$ sized invasive ductal carcinoma in her right breast. (A, B) MIBI scan demonstrated a focal uptake lesion in Right upper breast (arrows). (C, D) Mammography did not demonstrate any abnormal lesions in the heterogeneously dense breast. (E) Ultrasonography demonstrated an ill-defined irregular shape heterogenous lesion at 11 hours of the right breast, which was BI-RAD category 4 (dotted circle). MIBI, ${ }^{99 m} T$ Tc-methoxyisobutyl-isonitrile scintimammography; $\mathrm{RCC}$, right craniocaudal (CC) view; RMLO, right mediolateral oblique (MLO) view.
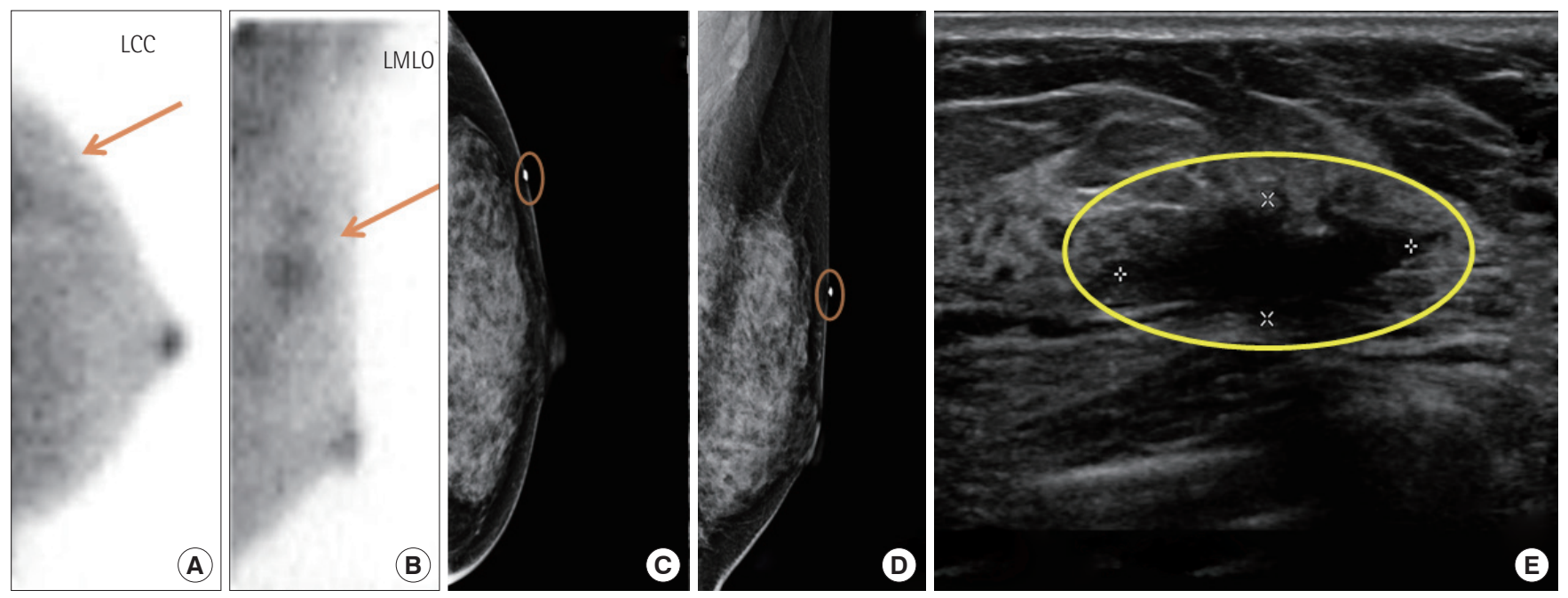

Fig. 2. MIBI scan negative result (benign lesion). Breast images of 42-year-old woman with biopsy-confirmed $24 \mathrm{~mm}$ fibrocystic change in left breast. (A, B) MIBI scan demonstrated benign lesion in Left upper breast (arrows). (C, D) Mammography did not demonstrate any abnormal lesions in heterogeneously dense breast. (E) Ultrasonography demonstrated ill-defined irregular shape hypoechoic lesion at 2 hours of left breast, which was BI-RAD category 4 (circle). MIBI, ${ }^{99 m}$ Tc-methoxyisobutyl-isonitrile scintimammography; RCC, right craniocaudal (CC) view; RMLO, right mediolateral oblique (MLO) view.

dictive value (59.1\%) would not have a diagnostic power over the general modalities due to significantly lower values compared to previous studies which reported their values ranging from $68 \%$ to $72 \%[13,14]$. Kim et al. [12] also reported a similar result that MIBI scan had better NPV compared with mammography in breast cancer patients with dense breast (84.8\% vs. 50.0\%). However, they enrolled all breast cancer patients with only consideration of breast density without any benign lesions.
There has been a recent resurgence in gamma imaging of the breast with the advancement gamma cameras in the past decade. Zhou et al. [17] reported that the development of a high-resolution, small field-of-view dedicated breast gamma imaging system has overcome 2 major limitations of gamma breast imaging (tumor localization and detection of sub-centimeter lesions) that previously prevented its wide adaptation. The authors also mentioned that breast gamma imaging is not influenced by breast density 

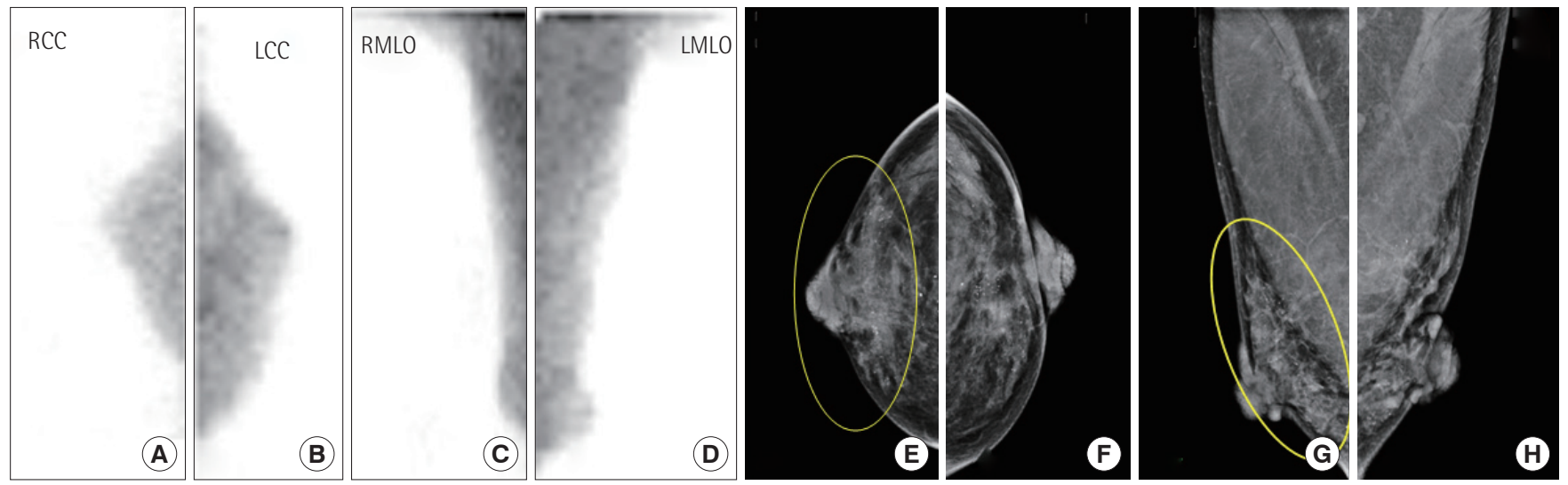

Fig. 3. MIBI scan negative result (benign lesion). Breast images of 25-year-old woman with a biopsy confirmed fibrocystic change and intraductal papilloma in both breast. (A-D) MIBI scan demonstrated a benign lesion in both breast. (E-H) Mammography demonstrate diffuse scattered microcalcifications lesions in both heterogeneously dense breast which was BI-RAD category 4 (circles). MIBI, ${ }^{99 m} \mathrm{Tc}-\mathrm{methoxy-}$ isobutyl-isonitrile scintimammography; RCC, right craniocaudal (CC) view; RMLO, right mediolateral oblique (MLO) view; LCC, left craniocaudal (CC) view.

similar to MRI and can obtain much improved specificity, even though it retained equivalent sensitivity as MRI [16-18].

Gamma breast imaging provides physiologic data in breast cancer imaging via 2 mechanisms. First, the radioactive tracer ${ }^{99 m} \mathrm{Tc}$ -sestamibi is distributed evenly throughout the circulatory system. Neo-angiogenesis occurs within the tumor microenvironments to support their increased proliferations. Thus, the pharmaceutical delivery to these lesions is enhanced [8]. Second, ${ }^{99 \mathrm{~m}} \mathrm{Tc}$-sestamibi binds mitochondria within tumor cells specifically. Due to a higher cytoplasmic mitochondrial density in the tumor cells comparing to the normal breast cells or benign tumor cells, ${ }^{99 \mathrm{~m}} \mathrm{Tc}$-sestamibi can better remain in the tumor cells [9]. These 2 mechanisms make gamma breast imaging highly sensitive and specific [18]. Therefore, MIBI scan shows the focal intensity uptake in the malignant lesions providing a more definite diagnosis (Fig. 1).

Although such a relationship plays an important role in the MIBI scan mechanism, this does not mean ${ }^{99 m} \mathrm{Tc}$-sestamibi does not bind with normal tissue. In patients with dense breast tissue, ${ }^{99 \mathrm{~m}} \mathrm{Tc}$-sestamibi could bind with normal breast tissue more intensely than in patients with fatty breast tissue. This may play a role in causing artifacts hindering tumor detection (Figs. 2, 3). This also might be a possible factor for the lower sensitivity of MIBI scan for younger women [14].

Hur et al. [13] commented that MIBI scintimammography might be one of the new possible modalities for preoperative evaluation compared to conventional images, because the high detection rate for malignant lesions in breasts was nearly equivalent to conventional imaging. However, the authors also mentioned that there was no superior benefit for multicentric or multifocal lesions or axillary metastasis compared to conventional imaging.
Contrary to the above reports, Edwards et al. [10] evaluated how often MIBI scan and MRI would influence surgical management in patients with breast cancer. The authors basically assumed that MIBI scan had greater sensitivity and specificity than standard imaging; its use should affect surgical management. They analyzed 163 patients divided into 2 groups; 118 had MIBI scan and 45 had MRI. The conversion rates of eligible BCS to mastectomy were $11.9 \%$ in MIBI scan to $28.9 \%$ in MRI, respectively. These results demonstrate that MIBI scan did not overestimate the extent of disease to which it would change surgical management. They concluded that MIBI scan is a useful adjunct in the surgical management of breast cancer that can be safely used in place of MRI [10].

Although we did not evaluate MRI in this study, it already has proved to have better sensitivity in discriminating breast cancer over other imaging modalities including ultrasonography, breast mammography, and MIBI scan [6,19]. However, MRI has a high false-positive rate in differentiating breast lesions in patients with breast cancers [20]. Therefore, the MIBI scan could be used to correctly diagnose benign lesions in women with suspicion of breast malignancy on MRI [15,16,19].

The diagnostic usefulness between MIBI scan and conventional imaging (i.e., ultrasonography/breast mammography) have been investigated and evaluated throughout many previous studies. In a direct comparison among these modalities, MIBI scan would provide a possibly "better" method to detect breast cancer that would be unveiled based on higher specificity and negative predictive values as in this study. In other words, MIBI scan can provide higher specificity and negative predictive values, meaning the negative results are benign lesions. Therefore, the 'real' diagnostic power for breast cancer over conventional imaging modalities could provide 
a confident differential diagnosis and help in avoiding any unnecessary procedures such as a fine-needle or core-needle biopsies.

This study has some limitations. First, this is a retrospective study that could lead to selection bias and a reduced statistical power. Second, we only enrolled patients with breast lesions without considering individual menstrual cycles that could affect the accuracy of conventional images. The radiotracer uptake in the breast could be influenced by hormonal changes throughout the cycle. Further prospective studies with a larger group are required to validate the diagnostic power of MIBI scan in comparison with the general modalities.

The mainstay diagnosis for breast cancers has been ultrasonography and breast mammography although other extensive imaging modalities including MR breast, positron emission tomography-computed tomography, and whole body bone scan could be used for the furtherer evaluations. BI-RAD category 4 or higher lesions have performed by needle biopsy rather than observation.

The usefulness of MIBI scan has been currently a controversial imaging technique that still requires further study and needs to be proven of its diagnostic values. Nonetheless, the results from this study have presented that MIBI scan might be one of the key modalities in detecting breast cancer along with conventional modalities.

There are some concerns of MIBI scan, such as the amount of radiation exposure, high expense, and others factors which might be an obstacle for some patients. These would be the disadvantages of MIBI scan as a routine screening modality.

MIBI scan may offer an alternative diagnostic tool for "invasive" biopsy procedures, because its superior specificity and negative predictive value provided confident results on non-proliferative benign lesions in this study. It also could be a good diagnostic modality for extremely dense breasts on mammography and post operative follow-up study for breast cancer patients, especially breast conserving surgery. Nonetheless, further investigation is necessary in those extremely dense cases or in postoperative follow-up.

\section{CONFLICT OF INTEREST}

No potential conflict of interest relevant to this article was reported.

\section{REFERENCES}

1. Korean Breast Cancer Society. Breast cancer facts \& figures 2015 [Internet]. Seoul: Korean Breast Cancer Society; 2015 [cited 2017 Jun 20]. Available from: http://www.kbcs.or.kr/sub02/sub04.html.

2. Teifke A, Hlawatsch A, Beier T, Werner Vomweg T, Schadmand S,
Schmidt M, et al. Undetected malignancies of the breast: dynamic contrast-enhanced MR imaging at 1.0 T. Radiology 2002;224:8818.

3. Potterton AJ, Peakman DJ, Young JR. Ultrasound demonstration of small breast cancers detected by mammographic screening. Clin Radiol 1994;49:808-13.

4. Rosenberg RD, Hunt WC, Williamson MR, Gilliland FD, Wiest PW, Kelsey CA, et al. Effects of age, breast density, ethnicity, and estrogen replacement therapy on screening mammographic sensitivity and cancer stage at diagnosis: review of 183,134 screening mammograms in Albuquerque, New Mexico. Radiology 1998; 209:511-8.

5. Kolb TM, Lichy J, Newhouse JH. Comparison of the performance of screening mammography, physical examination, and breast US and evaluation of factors that influence them: an analysis of 27,825 patient evaluations. Radiology 2002;225:165-75.

6. Berg WA, Gutierrez L, NessAiver MS, Carter WB, Bhargavan M, Lewis RS, et al. Diagnostic accuracy of mammography, clinical examination, US, and MR imaging in preoperative assessment of breast cancer. Radiology 2004;233:830-49.

7. Khalkhali I, Mena I, Jouanne E, Diggles L, Venegas R, Block J, et al. Prone scintimammography in patients with suspicion of carcinoma of the breast. J Am Coll Surg 1994;178:491-7.

8. Sharma S, Sharma MC, Sarkar C. Morphology of angiogenesis in human cancer: a conceptual overview, histoprognostic perspective and significance of neoangiogenesis. Histopathology 2005;46:4819.

9. Delmon-Moingeon LI, Piwnica-Worms D, Van den Abbeele AD, Holman BL, Davison A, Jones AG. Uptake of the cation hexakis(2-methoxyisobutylisonitrile)-technetium-99m by human carcinoma cell lines in vitro. Cancer Res 1990;50:2198-202.

10. Edwards C, Williams S, McSwain AP, Damle S, Rapelyea JA, Downs K, et al. Breast-specific gamma imaging influences surgical management in patients with breast cancer. Breast J 2013;19:512-9.

11. Kessler R, Sutcliffe JB, Bell L, Bradley YC, Anderson S, Banks KP. Negative predictive value of breast-specific gamma imaging in low suspicion breast lesions: a potential means for reducing benign biopsies. Breast J 2011;17:319-21.

12. Kim BS, Moon BI, Cha ES. A comparative study of breast-specific gamma imaging with the conventional imaging modality in breast cancer patients with dense breasts. Ann Nucl Med 2012;26:823-9.

13. Hur SM, Kim SH, Lee SK, Kim WW, Choi JH, Kim S, et al. The preoperative role of breast-specific gamma imaging for the breast cancer patients: in comparison with conventional imaging modality. J Breast Cancer 2010;13:198-205.

14. Lee A, Chang J, Lim W, Kim BS, Lee JE, Cha ES, et al. Effectiveness of breast-specific gamma imaging (BSGI) for breast cancer in Ko- 
rea: a comparative study. Breast J 2012;18:453-8

15. Brem RF, Floerke AC, Rapelyea JA, Teal C, Kelly T, Mathur V. Breast-specific gamma imaging as an adjunct imaging modality for the diagnosis of breast cancer. Radiology 2008;247:651-7.

16. Brem RF, Petrovitch I, Rapelyea JA, Young H, Teal C, Kelly T. Breast-specific gamma imaging with 99mTc-Sestamibi and magnetic resonance imaging in the diagnosis of breast cancer: a comparative study. Breast J 2007;13:465-9.

17. Zhou M, Johnson N, Gruner S, Ecklund GW, Meunier P, Bryn S, et al. Clinical utility of breast-specific gamma imaging for evaluating disease extent in the newly diagnosed breast cancer patient. Am J
Surg 2009;197:159-63.

18. Sampalis FS, Denis R, Picard D, Fleiszer D, Martin G, Nassif E, et al. International prospective evaluation of scintimammography with (99m)technetium sestamibi. Am J Surg 2003;185:544-9.

19. Kim BS. Usefulness of breast-specific gamma imaging as an adjunct modality in breast cancer patients with dense breast: a comparative study with MRI. Ann Nucl Med 2012;26:131-7.

20. Drew PJ, Turnbull LW, Chatterjee S, Read J, Carleton PJ, Fox JN, et al. Prospective comparison of standard triple assessment and dynamic magnetic resonance imaging of the breast for the evaluation of symptomatic breast lesions. Ann Surg 1999;230:680-5. 